Tohoku J. Exp. Med., 2004, 203, 353-357

Short Report

\title{
Supplementation of Antioxidants Prevents Oxidative Stress during A Deep Saturation Dive
}

\author{
Makoto Ikeda, Kazuhiko Nakabayashi, Masaharu Shinkai, Yukihiko Hara, ${ }^{1}$ \\ TAKAKo KIZAKI, ${ }^{2}$ SHUJI OH-ISHI ${ }^{3}$ and HideKI OHNO ${ }^{2}$ \\ Japan Maritime Self-Defense Force Undersea Medical Center, Yokosuka \\ 239-0826, \\ ${ }^{1}$ Food Research Laboratories, Mitsui Norin Co., Ltd., Fujieda 426-0023, \\ ${ }^{2}$ Department of Molecular Predictive Medicine and Sport Science, Kyorin Uni- \\ versity, School of Medicine, Mitaka 181-8611, and \\ ${ }^{3}$ Fifth Department of Internal Medicine, Tokyo Medical University, Inashiki \\ 300-0395
}

Ikeda, M., Nakabayashi, K., Shinkai, M., Hara, Y., Kizaki, T., OH-ishi, S. and Ohno, H. Supplementation of Antioxidants Prevents Oxidative Stress during A Deep Saturation Dive. Tohoku J. Exp. Med., 2004, 203 (4), 353-357 — Conflicting views exist at the present regarding the influences of a deep saturation dive on liver function in divers. Therefore, we first reevaluated whether a deep saturation dive (400 $\mathrm{msw}$ ) induces a hepatic disturbance. As the result, plasma activities of both transaminases (aspartate aminotransferase [AST] and alanine aminotransferase [ALT]) increased significantly, whereas cholinesterase (Ch-E) activity decreased markedly, being highly suggestive of liver dysfunction. Assuming that the liver dysfunction was attributable to oxidative stress, we next examined the effects of supplementation of antioxidants (600 $\mathrm{mg}$ of vitamin C, $150 \mathrm{mg}$ of $\alpha$-tocopherol, and $600 \mathrm{mg}$ of tea catechins per day) on liver function in saturation divers. As was anticipated, the antioxidants taken appeared to prevent a hepatic disturbance, indicating that a deep saturation dive provokes liver dysfunction probably due to oxidative stress. Thus, we recommend that saturation divers should take supplements of antioxidants. saturation dive; hyperbaric hyperoxia; liver function; oxidative stress; antioxidants (C) 2004 Tohoku University Medical Press

Received April 13, 2004; revision accepted for publication June 11, 2004.

Address for reprints: Hideki Ohno, M.D., Ph.D., Department of Molecular Predictive Medicine and Sport Science, Kyorin University, School of Medicine, 6-20-2 Sinkawa, Mitaka 181-8611, Japan. e-mail: ohnoh2o@kyorin-u.ac.jp 
Oxygen has been well known to be a doubleedged sword, since an epidemic of blindness in premature newborns was traced to retrolental fibroplasia induced by supplemental oxygen therapy. Thorsen et al. (2001) have indicated that the diving exposure is multifactorial including exposure to pressure per se, hyperoxia, and decompression stress associated with the formation of gas microemboli. The toxic effects of oxygen are also well known to be due mainly to its generation of reactive oxygen species (ROS). Thus, exposure to hyperoxia during a deep saturation dive is anticipated to cause oxidative damage to divers. Our previous studies have revealed that liver is more vulnerable than other organs to oxidative stress (Ohno et al. 1993; Nakanishi et al. 1995). Indeed, several investigators reported hyperbaric liver dysfunction in saturation divers (Philp et al. 1979; Doran et al. 1985), whereas the study by Thorsen et al. (2001) negated parenchymatous liver damage during a deep saturation dive. Therefore, in the current study, we first reevaluated whether a deep saturation dive induces a hepatic disturbance (Exp. 1). Because the findings obtained were highly suggestive of hepatic dysfunction, we next investigated the effects of antioxidant supplementation on hepatic function in saturation divers, assuming that the hepatic dysfunction was attributable, in large part, to oxidative stress (Exp. 2). The results show that the supplementation of antioxidants prevents a hepatic disturbance due to exposure to hyperoxia during a deep saturation dive.

\section{Materials ANd Methods}

Exp. 1 (the control group): Five healthy male uniformed divers (mean age: 36.2 years) participated in a $400 \mathrm{~m}$ of sea water (msw) (41 atmospheres absolute [ATA]) saturation dive without supplementation with antioxidants as a matter of duty. This mission was conducted using the Deep Diving Simulator at the Japan Maritime Self-Defense Force (JMSDF) Undersea Medical Center, Yokosuka. Informed consent was obtained from all participants including their families according to the Declaration of Helsinki, 1964 and Tokyo, 1975. The total time of the dive was 30 days. In both Exps. 1 and 2, the compression period to 41 ATA and the decompression period to 1 ATA were 38 hours 50 minutes and 17 days 14 hours 36 minutes, respectively. All divers were decompressed according to the Duke-GKSS decompression schedules (Vann 1984). $\mathrm{PO}_{2}$ in the chamber complex was kept at 0.42 ATA during the compression period and the stay at the bottom depth, and 0.50 ATA during the following decompression period, because of prophylaxis against decompression sickness.

Exp. 2 (the antioxidant group): Another five healthy male uniformed divers (mean age: 32.8 years) also participated in a $400 \mathrm{msw}$ saturation dive consuming antioxidants $(600 \mathrm{mg}$ of vitamin C, $150 \mathrm{mg}$ of $\alpha$-tocopherol, and $600 \mathrm{mg}$ of tea catechins [Mitui Norin Co., Ltd., Fujieda] per day) from three days prior to the dive to the day after surfacing. The antioxidants were orally taken three times a day after meals. The total time of the dive was 40 days.

As shown in Figs. 1 and 2, heparinized blood samples were collected by a corpsman from the antecubital vein in each experiment, and decompressed immediately through a service lock of the chamber complex. To avoid hemolysis, blood samples were decompressed from X ATA to $1 / 2 \mathrm{X}$ ATA at a rate of 2 ATA per min and kept at the pressure for $4 \mathrm{~min}$, and then the same procedure was repeated to reach the surface pressure.

Activities of aspartate aminotransferase (AST), alanine aminotransferase (ALT), and cholinesterase (Ch-E), as well as level of thiobarbituric reactive substances (TBARS) in plasma were determined using the respective commercial kits (Wako Pure Chemical Industries, Ltd., Osaka). Measurement of immunoreactive manganesesuperoxide dismutase (Mn-SOD) in plasma was made by an enzyme-linked immunosorbent assay, using a specific monoclonal antibody against Mn-SOD isoenzyme purified from human liver (Kawaguchi et al. 1990).

The statistical significance of the data was 
assessed by the Scheffé F-test. A 0.05 level of significance was used. Data are expressed as the mean \pm S.E.

\section{Results AND Discussion}

In Exp. 1, there were significant increases in the activities of both transaminases; that is, AST activity increased significantly by day 4 of exposure, and ALT activity also increased significantly on days 12, 30, and 37 (Fig. 1). In specialty, one diver showed a high activitiy of ALT over 100 IU/liter three times $(101,112$, and 107 IU/liter on days 12,30 , and 37 , respectively), with the preexposure activity being 32 IU/liter. In addition, plasma Ch-E activity decreased markedly on days 12,19 , and 25 . These findings were highly suggestive of liver dysfunction due to the saturation dive performed.

From the fact that a number of studies have indicated ROS generation due to the presence of excess oxygen at high-pressure levels (Philp et al. 1979; Doran et al. 1985; Somani and Husain 2000), we assumed that the hepatic disturbance observed in Exp. 1 resulted from oxidative stress. Therefore, we next examined the effects of antioxidant supplementation on liver function in saturation divers. As would be expected, there was no definite change in ALT activity, although one diver showed a high activity of the enzyme over $50 \mathrm{IU} /$ liter for this once (66 IU/liter on day 15) (Fig. 2A). Interestingly, on the other hand, AST activity reduced significantly by day 47 , but $\mathrm{Ch}-\mathrm{E}$ activity increased significantly on days 4 and 8 , although the physiological or pathological significance of these changes still remains obscure. From these findings, it seemed unlikely that the deep saturation dive provoked an apparent hepatic disturbance due to oxidative stress in Exp. 2. Indeed, plasma values of TBARS, which are used most as an index of oxidative stress, did not rise, but, on the contrary, decreased significantly on days 21, 40, and 47 (Fig. 2B), although the physiological meaning also remains to be elucidated. Mn-SOD also provides a marker of oxidative stress, because this SOD isoenzyme is induced by increased exposure to superoxide radicals (Ohno et al. 1993). Likewise, plasma Mn-SOD level did not increase substantially in Exp. 2. Collectively, the results obtained in the current study suggest that a deep saturation dive induces a hepatic disturbance probably owing to oxidative stress.

In the current study, we have revealed that supplementatiom of antioxidants can prevent a hepatic disturbance in saturation divers, thereby suggesting that synergistic effects of vitamin C, vitamin $\mathrm{E}$, and tea catechins, which are all well

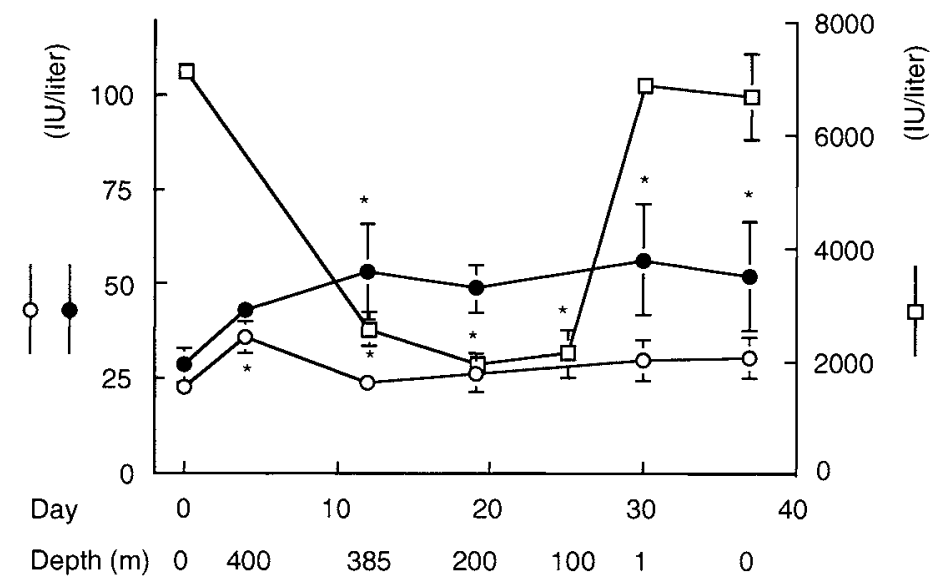

Fig. 1. Effects of a $400 \mathrm{~m}$ saturation dive on activities of three liver function-related enzymes in plasma from five divers. Values are mean \pm s.E. AST, aspartate aminotransferase; ALT, alanine aminotransferase; Ch-E, cholinesterase. " $p<0.05$ vs. "day 0" values.

$\bigcirc$, AST; O, ALT; $\square$, Ch-E. 


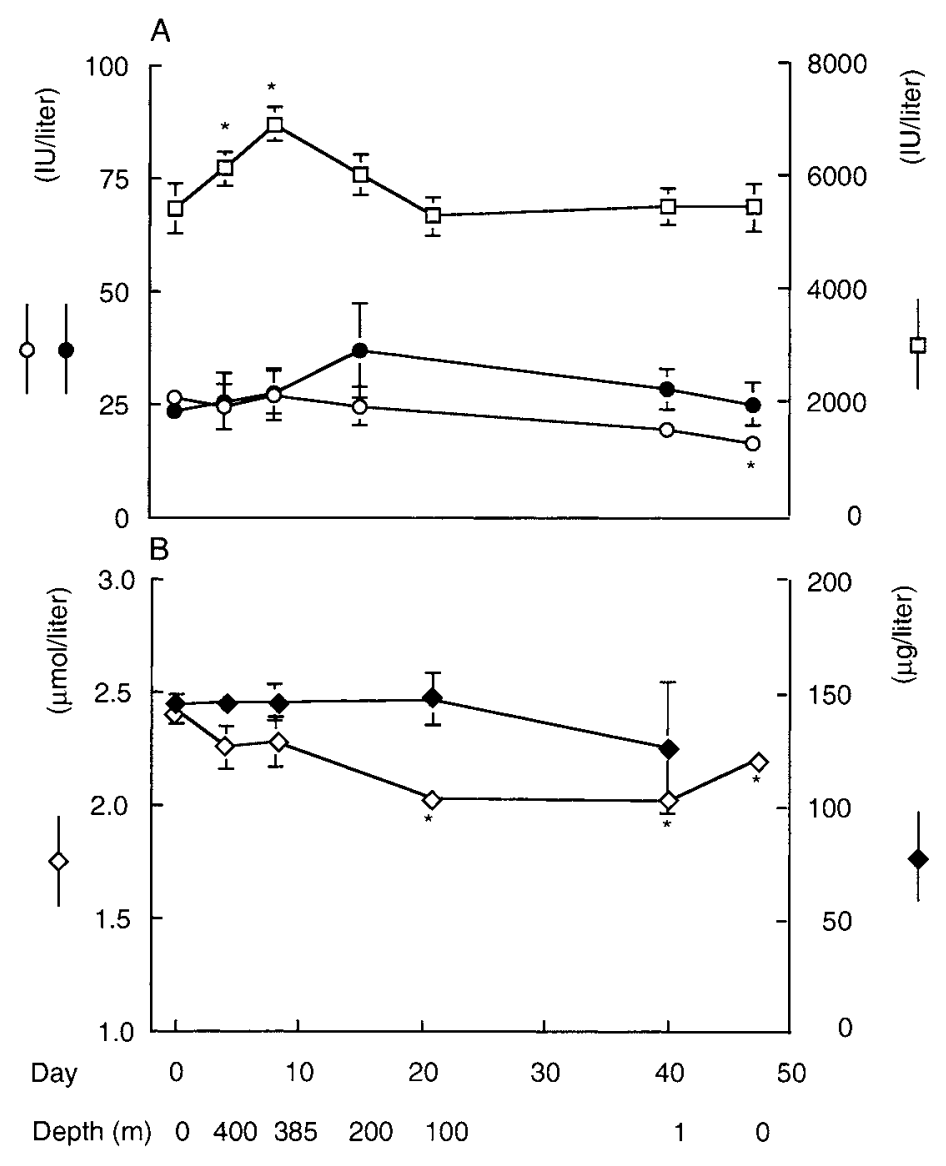

Fig. 2. Effects of a $400 \mathrm{~m}$ saturation dive on activities of three liver function-related enzymes (A) and levels of two markers of oxidative stress (B) in plasma from five divers receiving antioxidant supplements. Values are mean \pm S.E. TBARS, thiobarbituric reactive substances; Mn-SOD, manganesesuperoxide dismutase. " $p<0.05$ vs. "day 0 " values.

O, AST; ๑, ALT; $\square$, Ch-E; $\diamond$, TBARS; $\diamond$, Mn-SOD.

known as efficacious antioxidants and easily available (Decker and Clarkson 2000), upon oxidative stress are expected under hyperbaric hyperoxia. Although the precise mechanism by which oxidative stress induces liver dysfunction during a deep saturation dive is not clear, we recommend that saturation divers should take supplements of antioxidants. Moreover, supplementation of antioxidants may also be beneficial to health in recreational divers.

\section{Acknowledements}

The authors thank Drs. S. Suzuki, H. Yamaguchi, H. Miyazaki, and M. Segawa for their kind cooperation.

\section{References}

Decker, E.A. \& Clarkson, P.M. (2000) Dietary sources and bioavailability of essential and nonessential antioxidants. In: Handbook of Oxidants and Antioxidants in Exercise, edited by C.K. Sen, L. Packer \& O. Hänninen, Elsevier, Amsterdam, pp. 323-358.

Doran, G.R., Chaudry, L., Brubakk, A.O. \& Garrard, M.P. (1985) Hyperbaric liver dysfunction in saturation divers. Undersea Biomed. Res., 12, 151-164.

Kawaguchi, T., Suzuki, K., Matsuda, Y., Nishimura, T., Uda, T., Ono, M., Sekiya, C., Ishikawa, M., Iino, S., Endo, Y. \& Taniguchi, N. (1990) Serum-manganese-superoxide dismutase: normal values and increased levels in patients with acute myocardial infarction and several malig- 
nant diseases determined by an enzyme-linked immunosorbent assay using a monoclonal antibody. J. Immunol. Methods, 127, 249-254.

Nakanishi, K., Tajima, F., Nakamura, A., Yagura, S., Ookawara, T., Yamashita, H., Suzuki, K., Taniguchi, N. \& Ohno, H. (1995) Effects of hypobaric hypoxia on antioxidant enzymes in rats. J. Physiol., 489, 869-876.

Ohno, H., Kayashima, S., Nagata, N., Yamashita, H., Ookawara, T. \& Taniguchi, N. (1993) Changes in immunoreactive manganese-superoxide dismutase concentration in human serum after $93 \mathrm{~h}$ strenuous physical exercise. Clin. Chim. Acta, 215, 213-219.

Philp, R.B., Bennett, P.B., Andersen, J.C., Fields, G.N., Mcintyre, B.A., Francey, I. \& Briner, W. (1979) Effects of aspirin and dipyridamole on platelet function, hematology, and blood chemistry of saturation divers. Undersea Biomed. Res., 6, 127-146.

Somani, S.M. \& Husain, K. (2000) Influence of exercise-induced oxidative stress on the central nervous system. In: Handbook of Oxidants and Antioxidants in Exercise, edited by C.K. Sen, L. Packer \& O. Hänninen, Elsevier, Amsterdam, pp. 713-751.

Thorsen, E., Haave, H., Hofso, D. \& Ulvik, R.J. (2001) Exposure to hyperoxia in diving and hyperbaric medicine-effects on blood cell counts and serum ferritin. Undersea Hyper. Med., 28, 57-62.

Vann, R.D. (1984) Decompression from saturation diving. In: Proc. of the 3rd. Annual Canadian Ocean Technology Conference, edited by D.R. Rosser, Underwater Canada, Toronto, pp. 175-186. 